\title{
Traitement de l'œstrose ovine par injection d'un insecticide organophosphoré le Diméthoate
}

\author{
par G. UILENBERG $(*)$, A. PERDRIX $(* *)$ et P. DUBOIS $(* *)$
}

\begin{abstract}
RESUME
Le diméthoate, injecté par voie sous-cutanée, n'est pas dangereux pour les moutons au repos et abrités de la chaleur du soleil, à la dose de $30 \mathrm{mg} / \mathrm{kg}$. Il est efficace, à $25 \mathrm{mg} / \mathrm{kg}$, contre les larves d'Oestrus ovis. La chaleur et la fatigue semblent constituer des contre-indications.
\end{abstract}

La sinusite parasitaire des petits ruminants, causée par les larves d'Oestrus ovis Linné, 1761 (Oestridae, Diptera) est très répandue à Madagascar et y cause des pertes économiques importantes; il est rare que l'autopsie d'un mouton pris au hasard n'en révèle pas.

Jusqu'à l'arrivée des insecticides systémiques le traitement était fastidieux ou inefficace. Actuellement, certains de ces produits administrés per os peuvent atteindre les larves par l'intermédiaire de la circulation sanguine. Les premiers à avoir expérimenté un traitement systémique par injection parentérale, méthode plus pratique que la précédente, sont, à notre connaissance, PETERSON et al. (1959) aux Etats-Unis. Ils estiment que le diméthoate, administré par voie intramusculaire sous forme d'une solution injectable contenant 50 p. 100 d'insecticide, n'est pas dangereux pour les moutons à la dose de $25 \mathrm{mg} / \mathrm{kg}$ de poids vif et tue 97 p. 100 des larves $(98$ p. 100 de celles du premier stade, 97 p. $100 \mathrm{du}$ deuxième et 92 p. $100 \mathrm{du}$ troisième).

Les conditions de l'élevage des ovins à Madagascar sont notablement différentes de celles

(*) Institut d'Elevage et de Médecine vétérinaire des Pays tropicaux, 10, rue Pierre Curie, 94 - MaisonsAlfort, France.

$(* *)$ Institut d'Elevage et de Médecine vétérinaire des Pays tropicaux, Région de Recherches de Madagascar, B.P. 4, Tananarive, Madagascar. rencontrées aux U.S.A. Dans l'lle cet élevage est extensif dans la mesure où les animaux sont pratiquement abandonnés à eux-mêmes pour la recherche de leur alimentation et de leur boisson, avec une alternánce marquée d'abondance et de disette. Le polyparasitisme y est fréquent et intense. Aussi la plupart des animaux présentent-ils un état général peu satisfaisant, ce qui nous a paru susceptible de les rendre particulièrement sensibles à de possibles qualités toxiques du produit.

Nous avons donc effectué des recherches précises à cet égard, complétées par des observations portant sur son efficacité, le tout, précisons-le à nouveau, dans les conditions propres à Madagascar - ce qui en rend les conclusions valables partout où, sous les tropiques, l'élevage ovin est soumis aux mêmes conditions écologiques et nosologiques.

Nous avons voulu déterminer les séquelles toxiques de ce traitement dans les conditions de Madagascar et vérifier son efficacité sur l'œstrose des ovins malgaches.

\section{MATERIEL ET METHODES}

Trois présentations de diméthoate ont été expérimentées :

a) Des liquides émulsionnables contenant 
40 et 50 p. 100 de diméthoate, injectés par voie intramusculaire ou par voie sous-cutanée ("3).

b) Une émulsion contenant 2 p. 100 de diméthoate, faite à partir du liquide émulsionnable à 50 p. 100 par dilution dans l'eau physiologique, injectée par voie intramusculaire ou par voie sous-cutanée. Cette forme a été essayée, afin d'éviter l'emploi des très faibles quantités injectées en utilisant le liquide non dilué (souvent $1 \mathrm{ml}$ ou moins); la précision nécessaire est alors trop grande pour les conditions de brousse.

c) Une solution de diméthoate technique dans de l'eau physiologique à 2 p. 100 , injectée par voie sous-cutanée. Le diméthoate technique se dissout, à la température ambiante du laboratoire, complètement à 2 p. 100 en moins de $24 \mathrm{~h}$, mais une solution à 2,5 p. 100 n'a pas pu être obtenue.

Les expériences de toxicité au laboratoire ont porté sur 71 moutons, dont 18 de race locale (à poils) et 53 mérinos d'Arles, de divers âges (de quelques mois à plus de 4 ans), en bonne ou assez bonne condition, hormis quelques animaux de race locale, très maigres. Ces animaux ont été gardés avant et après les injections à l'abri du soleil et au repos. Les doses inoculées ont été de 25 et $30 \mathrm{mg}$ de diméthoate par $\mathrm{kg}$. (Les doses sont en réalité plus élevées pour les mérinos si l'on tient compte du poids de la toison, environ 5 à presque 10 p. $100 \mathrm{du}$ poids total.) Les moutons ont été pesés individuellement.

Un de nos confrères a signalé une mortalité importante après traitement à $25 \mathrm{mg} / \mathrm{kg}$ chez des moutons de race locale, dans la région de Majunga. Le produit injecté était le liquide émulsionnable à 40 p. 100 , non dilué, administré par voie sous-cutanée. Les animaux étaient exposés au soleil et ils étaient en outre fatigués par une longue marche avant et après l'injection. Pour cette raison, nous avons également fait une expérience de toxicité dans la même région à climat chaud : 20 moutons de race locale, maigres ou en condition moyenne, ont reçu, après pesée individuelle, par voie sous-cutanée, $25 \mathrm{mg} / \mathrm{kg}$ de liquide émulsionnable à 40 p. 100 , non dilué. Les animaux ont été gardés en plein soleil, mais ils étaient au

(3) Deux présentations commerciales, le Rogor-L 40 et le Rogor-L 50, de la Maison Montecatini Edison. repos depuis une semaine avant l'injection; celle-ci a été suivie d'une période d'observation (et de repos) de quatre jours. Les injections ont été faites à $11 \mathrm{~h}$ du matin, par temps chaud.

L'expérience sur l'efficacité du traitement a été limitée; nous avons voulu vérifier si les bons résultats signalés par PETERSON et al. sont également valables contre l'œstrose à Madagascar. Neuf moutons maigres, de race locale, porteurs d'œstres, ont été achetés. Cinq d'entre eux ont été traités avec la dose de $25 \mathrm{mg} / \mathrm{kg}$ (émulsion à 2 p. 100 , par voie souscutanée); les 4 autres ont servi comme témoins. Tous ces animaux ont été autopsiés 3 à 5 jours après le traitement; les sinus et passages nasaux ont été soigneusement inspectés, en déroulant les os turbinés.

Les injections par voie sous-cutanée ont été faites sous la peau de la paroi thoracique (l'endroit le plus commode chez les mérinos se situe juste en dessous de la limite de la laine, près de l'aisselle). Les injections par voie intramusculaire ont été faites dans les muscles de la cuisse.

\section{RESULTATS}

\section{Toxicité générale}

\section{a) Expériences au laboratoire}

Aucun des moutons n'est mort par intoxication. Aucun des moutons inoculés avec $25 \mathrm{mg} / \mathrm{kg}$ (12 sujets) n'a montré de symptômes d'intoxication. Parmi les 59 animaux inoculés avec $30 \mathrm{mg} / \mathrm{kg}$, plusieurs mérinos présentaient une respiration accélérée pendant un jour et une hyperthermie pendant quelques jours. Quatre mérinos et un mouton local ont en outre présenté une hypersalivation pendant 1 ou 2 jours; un d'entre eux a eu pendant un jour des mouvements incoordonnés de la tête, un autre a présenté pendant un jour un écoulement séreux des narines, des tremblements des muscles du menton pendant deux jours et il a présenté une respiration accélérée et de l'ąbattement pendant trois jours.

\section{b) Expérience à Majunga}

Un sujet est mort au bout de trois jours, après avoir présenté dès le premier jour inappétence, dyspnée, raideur de l'arrière-train et diarrhée. A l'autopsie on a constaté un œdème pulmonaire, des suffusions sanguines sur le cour et un hydropéricarde sanguinolent. Les 19 autres animaux ont guéri. 
A l'opposé de ce que nous avions observé au laboratoire, une diarrhée est apparue sur l'ensemble du troupeau dans les 3 à $4 \mathrm{~h}$ suivant l'injection; elle a persisté chez certains sujets pendant trois jours. On n'a pas observé d'hypersalivation.

La plupart des animaux ont présenté une raideur de l'arrière-train, qui a persisté chez certains le lendemain du traitement.

Quatre sujets ont présenté de l'inappétence pendant un à trois jours; deux de ces animaux avaient en outre une dyspnée qui a disparu le lendemain de l'injection.

Un sujet a présenté une heure après le traitement, un odème de la tête; cet odème avait totalement disparu au bout de deux jours.

\section{Réactions locales}

L'injection par voie intramusculaire avec le liquide émulsionnable, dilué ou pur, détermine souvent une boiterie pendant plusieurs jours, et chez quelques animaux sacrifiés, il y avait parfois une nécrose locale et des hémorragies au lieu d'injection.

L'injection par voie sous-cutanée avec l'émulsion à 2 p. 100 donne lieu chez la plupart des animaux à un cedème chaud et douloureux. L'autopsie de plusieurs sujets sacrifiés a montré une infiltration gélatineuse, pouvant atteindre un diamètre de plus de $10 \mathrm{~cm}$ et une épaisseur de 2 à $3 \mathrm{~cm}$. L'œdème disparaît graduellement après une dizaine de jours.

Le liquide émulsionnable non dilué, injecté par voie sous-cutanée, détermine également un tel œdème, mais il est beaucoup moins important.

Finalement, la solution de diméthoate technique donne le moins de réaction locale (seule la voie sous-cutanée a été expérimentée); un léger odème peut exister.

\section{Efficacité}

Les 4 témoins (choisis au hasard parmi les 9 sujets) avaient un total de 71 larves vivantes d'Oestrus ovis, dont $53 \mathrm{du}$ premier stade, $10 \mathrm{du}$ deuxième et 8 du troisième; aucune larve morte. 2 des sujets avaient une sinusite importante, un autre une sinusite légère.

Les 5 moutons traités avaient un total de 5 larves vivantes (aucune du premier stade, 4 du deuxième et une du troisième); en plus il y avait 5 larves mortes, dont une du premier stade, $3 \mathrm{du}$ deuxième et une du troisième. Un des sujets présentait une sinusite importante, 3 autres une sinusite légère.

\section{DISCUSSION ET CONCLUSION}

Au laboratoire aucune intoxication dangereuse n'a suivi l'injection de diméthoate aux doses de 25 et $30 \mathrm{mg} / \mathrm{kg}$. Cela correspond aux données de la bibliographie; par exemple PETERSON et al. (1959) trouvent que les moutons en bon état supportent sans troubles des doses jusqu'à $80 \mathrm{mg} / \mathrm{kg}$; ils observent quelques réactions toxiques (non mortelles) chez des animaux en mauvaise condition à partir de $30 \mathrm{mg} / \mathrm{kg}$, réactions que nous avons pu observer à $30 \mathrm{mg} / \mathrm{kg}$ même chez des animaux en bon état; aucune intoxication à $25 \mathrm{mg} / \mathrm{kg}$ dans nos expériences.

Les moutons exposés à la chaleur semblent beaucoup plus sensibles, étant donné les résultats obtenus à Majunga. La fatigue pourrait également intervenir, étant donné la mortalité importante observée par un confrère à Majunga chez des animaux fatigués et exposés au soleil. Il ne faut donc traiter que des moutons au repos et abrités du soleil.

La réaction locale est négligeable après injection par voie sous-cutanée d'une solution de diméthoate technique; le liquide émulsionnable pur, injecté par voie sous-cutanée, ne donne pas lieu non plus à une réaction de quelque importance. Par contre, le liquide émulsionnable pur ou dilué est à déconseiller par voie intramusculaire, tandis que la réaction locale est souvent importante après injection par voie sous-cutanée de l'émulsion.

La préférence semble devoir aller à la solution de diméthoate technique, exempt d'adjuvants utilisés par le fabriquant et dont la composition n'est pas divulguée.

L'efficacité semble totale contre les larves du premier stade, à $25 \mathrm{mg} / \mathrm{kg}$; elle est moins bonne, mais réelle, contre les autres stades, mais le faible nombre de larves du deuxième et troisième stades chez les témoins ne permet pas de la chiffrer. La sinusite n'a pas disparu 5 jours après le traitement.

Le prix du traitement est modique et ne 
dépasse pas, dans les conditions actuelles à Madagascar, 0,05 francs français par mouton (en ne comptant évidemment que l'insecticide). Le fait que la chaleur et la fatigue constituent des contre-indications, et que le poids indivi- duel des animaux doit être connu avec une assez grande précision, nécessitera une grande prudence lors de son application sur le terrain à Madagascar et partout ailleurs où se présenteront les mêmes difficultés.

\section{SUMMARY}

Treatment of Oestrus ovis infestation in sheep by injection of an organophosphorus insecticide, dimethoate

Dimethoate, injected subcutaneously, is not dangerous at the dose of $30 \mathrm{mg} / \mathrm{kg}$ for sheep at rest and protected from the hot sun. It is active, at $25 \mathrm{mg} / \mathrm{kg}$, against larvae of Oestrus ovis. Its use is contra-indicated in hot weather and in tired animals.

\section{RESUMEN}

Tratamiento de la infestación por Oestrus ovis de la oveja por inyección de un insecticida organofosforado el Dimethoate

El Dimethoate, inyectado por via subcutánea, no es peligroso para las ovejas descansadas y amparadas del calor del sol, en dosis de $30 \mathrm{mg} / \mathrm{kg}$. La dosis de $25 \mathrm{mg} / \mathrm{kg}$ es eficaz contra las larvas de Oestrus ovis. El calor y el cansancio parecen constituir contraindicaciones.

\section{BIBLIOGRAPHIE}

PETERSON (H. O.), COBBETT (N. G.) et MELE-

NEY (W. P.), "Treatment of Oestrus ovis with dimethoate », Vet. Med., 1959, 54, 377-383. 